Draft version OCtober 30, 2018

Preprint typeset using $\mathrm{IAT}_{\mathrm{E}} \mathrm{X}$ style emulateapj v. 5/2/11

\title{
THE TRIPLE EVOLUTION DYNAMICAL INSTABILITY: STELLAR COLLISIONS IN THE FIELD AND THE FORMATION OF EXOTIC BINARIES
}

\author{
Hagai B. Perets ${ }^{1,2}$ \& Kaitlin M. Kratter ${ }^{2}$ \\ 1 Deloro Fellow, Technion - Israel Institute of Technology, Haifa, Israel 32000 \\ 2 Harvard-Smithsonian Center for Astrophysics, 60 Garden St.; Cambridge, MA, USA 02138 \\ Draft version October 30, 2018
}

\begin{abstract}
Physical collisions and close approaches between stars play an important role in the formation of exotic stellar systems. Standard theories suggest that collisions are rare, occurring only via random encounters between stars in dense clusters. We present a different formation pathway, the triple evolution dynamical instability (TEDI), in which mass loss in an evolving triple star system causes orbital instability. The subsequent chaotic orbital evolution of the stars triggers close encounters, collisions, exchanges between the stellar components, and the dynamical formation of eccentric compact binaries (including Sirius like binaries). We demonstrate that the rate of stellar collisions due to the TEDI is approximately $10^{-4} \mathrm{yr}^{-1}$ per Milky-Way Galaxy, which is nearly 30 times higher than the total collision rate due to random encounters in the Galactic globular clusters. Moreover, we find that the dominant type of stellar collisions is qualitatively different; most collisions involve asymptotic giant branch stars, rather than main sequence, or slightly evolved stars, which dominate collisions in globular clusters. The TEDI mechanism should lead us to revise our understanding of collisions and the formation of compact, eccentric binaries in the field.
\end{abstract}

\section{INTRODUCTION}

The predominant cause of stellar collisions is thought to be random encounters between stars in dense clusters. These collisions, where stars pass within a single stellar radius of each other, may result in a wide range of outcomes, most notably the formation of close and eccentric compact binaries (Shara 1999, 2002; Davies 2002; Ivanova 2010, and references therein). Because the collision rate per star, $\Gamma$, due to random encounters depends directly on the local number density of stars $\Gamma \propto n$, such interactions are common only in the densest stellar environments such as globular clusters (GCs). Outside of these clusters, the densities are lower by a factor as great as $10^{7}$ : random collisions effectively do not occur in the field. In this paper, we describe a dynamical instability that occurs in evolved systems containing three or more start 1 . This mechanism, which we term the triple evolution dynamical instability (hereinafter TEDI), leads to close encounters and collisions between stars even in low density stellar environments.

The TEDI occurs due to an interplay between stellar mass loss and orbital instability. Consider a hierarchical triple system composed of an inner binary coupled to an outer binary, where the outer binary consists of a tertiary companion in orbit about the center of mass of the inner pair. When the orbits of the inner and outer pair are well separated, the system can remain stable indefinitely. Stellar evolution can disrupt this architecture. When the more massive component of the inner binary evolves, it begins to shed mass. As a result, the orbits expand in proportion to the ratio between the initial and final mass in the enclosed system (Hadjidemetriou 1963; Eggleton 2006). Since the relative mass loss in the inner binary is greater than that in the outer binary, the inner orbit

\footnotetext{
${ }^{1}$ We focus here on triple systems, but the scenario is also applicable to higher multiplicity systems.
}

encroaches on the outer orbit. This relative orbital expansion can trigger a dynamical instability, which results in the dissolution of the system (see Fig. 1).

The subsequent evolution of such unstable systems is very similar to that of binary-single star encounters in globular clusters, and often leads to close encounters between any of the three components. The TEDI provides a unique pathway to physical collisions during these close approaches: as stars evolve off of the main sequence, they not only lose mass, but also expand radially by a factor of a few hundred on the Asymptotic Giant Branch (AGB). The simultaneity of mass loss (and thus instability) with stellar bloating greatly increases the cross section for collisions. For this reason, as we now demonstrate, the TEDI is the dominant route to stellar collisions in the Universe, producing nearly 30 times more collisions than random gravitational encounters in GCs.

The significance of mass-loss induced instability in triple systems has been proposed in several studies (Kiseleva et al. 1994; Iben \& Tutukov 1999; Perets 2010; Freire et al. 2011; Portegies Zwart et al. 2011). However, the frequency of this phenomena, and its importance for stellar collisions and the consequences thereof, have not been explored.

Our primary goal in this paper is to estimate the rate of collisions per galaxy induced by the TEDI mechanism, $\Gamma_{\mathrm{col}}$. This calculation requires the estimation of the following quantities:

- $N_{\star}$ - The number of stars in the Galaxy

- $f_{\text {evolve }}$ - The observed fraction of evolved stars

- $f_{\text {triple }}$ - The fraction of stars in hierarchical triple systems

- $f_{\text {des }}$ - The fraction of hierarchical systems that become unstable as the stars evolve 
- $f_{\text {col }}$ - The fraction of unstable triple systems that end with a collision between two of the stars

Thus $\Gamma_{\text {col }}=N_{\star} \times f_{\text {evolve }} \times f_{\text {triple }} \times f_{\text {des }} \times f_{\text {col }}$.

Observations provide estimates of the first two variables, and less certain estimates for the third (triple fraction). Attaining estimates for $f_{\text {des }}$ and $f_{\text {col }}$ occupies the remainder of this paper, as well as addressing the uncertainties in the triple fraction, $f_{\text {triple. The latter is }}$ done with both observational estimates and theoretical population synthesis models to provide independent estimates. The main difficulties in doing so are due to the limited data existing on triple systems and the distribution of their properties, and the large phase space of possible evolutionary routes. Given these caveats, we can only hope to provide an order of magnitude estimate of the importance of the TEDI. Though we provide the formal statistical uncertainties for the resulting fractions we obtain, the systematic uncertainties due to our limited knowledge of the distribution of triple system properties are likely larger than the statistical ones.

The outline of the paper is as follows. In Section 2 we quantify the criterion for the TEDI. We then determine the fraction of systems that undergo the TEDI, $f_{\text {des }}$, in Section 3, followed by an estimate of the frequency, $f_{\text {col }}$, of collisions in the destabilized systems (\$4). In Section 5 we use these results to provide an estimate of the total collision rate in the galaxy. We describe the various consequences of the TEDI in Section 6, and briefly point out the influence of secular dynamics on triple stellar evolution (8). We close the paper with the discussion and summary in Section 9 .

\section{THE TRIPLE EVOLUTION DYNAMICAL INSTABILITY} (TEDI)

A triple system is dynamically unstable when $Q<Q_{\text {st }}$, where $Q=a_{\text {out }}\left(1-e_{\text {out }}\right) / a_{\text {in }}$ and $Q_{\text {st }}$ is (we adopt Valtonen et al. 2008; but see also Mardling 2008 for a different definition) :

$Q_{\mathrm{st}}=3\left(1+m_{3} / M_{12}\right)^{1 / 3}\left(1-e_{\mathrm{out}}\right)^{-1 / 6}\left(\frac{7}{4}+\frac{1}{2} \cos i-\cos ^{2} i\right)^{1 / 3}, \frac{a_{\mathrm{in}, \mathrm{f}} / a_{\mathrm{out}, \mathrm{f}}}{a_{\mathrm{in}, \mathrm{i}} / a_{\mathrm{out}, \mathrm{i}}}=\left(\frac{m_{1, \mathrm{i}}+m_{2}}{m_{1, \mathrm{f}}+m_{2}}\right)\left(\frac{m_{1, \mathrm{f}}+m_{2}+m_{3}}{m_{1, \mathrm{i}}+m_{2}+m_{3}}\right)$.

where $a_{\text {in }}$ and $a_{\text {out }}$ are the semi-major axes (SMAs) of the inner and outer binaries in the triple system, $e_{\text {out }}$ is the outer binary eccentricity, and $m_{3}$ and $M_{12}=m_{1}+m_{2}$, are the masses of the outer component and the inner binary, respectively. The mutual inclination between the inner and outer binaries is $i$.

As the primary (with mass $m_{1}$ ) in the inner binary evolves off the main sequence (MS), it loses mass. We consider only systems where the stellar-wind driven mass loss occurs adiabatically, i.e. on a timescale that is long compared to the outer orbital period (prompt mass loss could occur in supernovae and also lead to instability) 2 .

\footnotetext{
2 The triple supernova instability scenario is limited only to high mass systems. Moreover, in the majority of cases, supernova explosions lead to the prompt unbinding of the system (especially if they are accompanied by a natal kick), making longer term chaotic evolution, and hence physical collisions, less likely than in the case of adiabatic mass loss. The contribution of this process to the over all collision rate in the Galaxy is therefore likely to be small, and not affect our main results. Nevertheless, this process, and the effects of natal kicks, could be especially important for collisions with neutron stars and black holes, and merits further study.
}

We further assume that mass loss is isotropic, and that mass transfer is negligible. The expansion of the inner orbit due to mass loss will be larger than the expansion of the outer orbit because the relative mass loss in the inner system is larger. Thus the SMAs evolve as:

$$
\frac{a_{\text {in }, \mathrm{f}}}{a_{\mathrm{in}, \mathrm{i}}}=\frac{m_{1, \mathrm{i}}+m_{2}}{m_{1, \mathrm{f}}+m_{2}}>\frac{m_{1, \mathrm{i}}+m_{2}+m_{3}}{m_{1, \mathrm{f}}+m_{2}+m_{3}}=\frac{a_{\mathrm{out}, \mathrm{f}}}{a_{\mathrm{out}, \mathrm{i}}}
$$

(Hadjidemetriou 1963) where the subscripts correspond to the system components and the evolutionary stage of the system ( $i$ and $f$ for the initial and the final, post-mass loss system, respectively). The expansion of each of the SMAs in direct proportion to the mass loss derives from the conservation of the quantity $G M a\left(1-e^{2}\right)$, where $M$ is the total system mass. Note that slow, isotropic mass loss is required for $e$ to remain constant.

The ratio between the SMAs of the outer and inner binaries therefore increases, by a factor of
The TEDI occurs when this increase drives a stable triple system across the threshold defined in Eq. (1). The subsequent dynamical evolution of an unstable system is chaotic. The system may evolve through various configurations, including exchanges between the stars in the inner and outer binaries, eccentricity excitation, and collisions (see Fig. 11 for an example). The final outcome is typically the ejection of one star and/or a physical collision. Because this three body problem is genuinely chaotic (typical Lyapunov exponent of 1/2; Ivanov \& Chernin 1991; Heinämäki et al. 1999; Valtonen \& Karttunen 2006 and references therein), the outcome is strongly dependent on small variations in the initial conditions. 

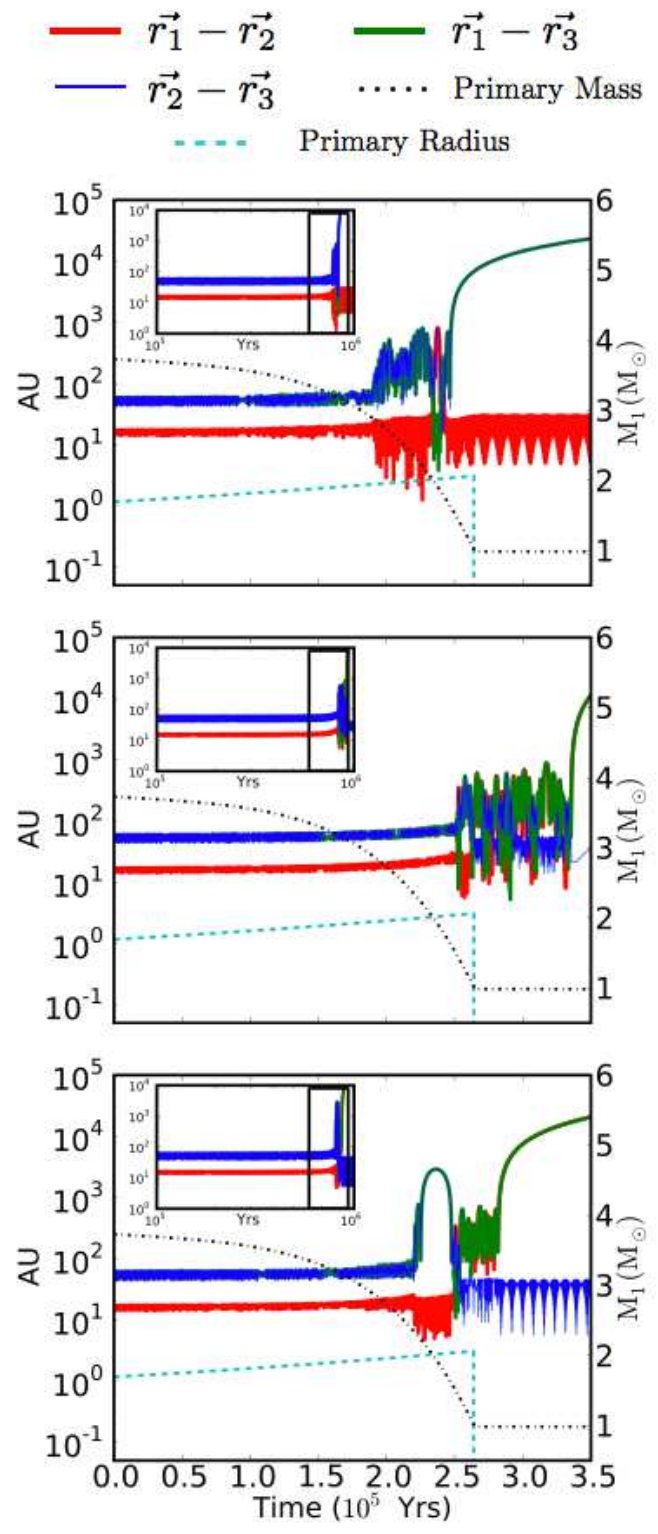

FIG. 1.- Three-body simulations of the dynamical evolution of triple systems undergoing adiabatic mass loss. The separations $(r)$ between each of the three components in each system are plotted as a function of time. Also shown are the mass (dashed-dotted) and the stellar radius (dashed) of the primary, $M_{1}$. Thick lines show the pairs including the primary. Top (1a): A collision between a MS star and an AGB star (the subsequent evolution, assuming point masses and no collision is also shown). Middle (1b): the formation of an eccentric close WD-MS binary, similar to the Sirius system. Bottom (1c): the WD primary is ejected after several exchanges, leaving behind an eccentric MS-MS binary. In all cases the systems started with masses of $M_{1}=5 M_{\odot}, M_{2}=2.0 M_{\odot}, M_{3}=$ $2.25 M_{\odot}, a_{\text {in }}=15 \mathrm{AU}, a_{\text {out }}=53 \mathrm{AU}, \mathrm{e}_{\text {out }}=0.1, \mathrm{i}=0.1$.

\section{ESTIMATE OF THE FRACTION OF SYSTEMS DESTABILIZED DUE TO THE TEDI}

To determine whether a given system is unstable, we simultaneously follow its orbital and stellar evolution, and check whether or not it violates the stability criterion at any point during its evolution. We estimate the fraction of destabilized systems in two ways: (1) we determine the fraction of observed triple systems (Tokovinin 2008) with known orbital parameters that become unstable, and (2) we calculate the unstable fraction of a synthetic population of triples modeled on binary statistics to account for potential biases in the observed sample.

We first describe the procedure to identify systems that should become unstable, and then calculate the instability rates for the observed and the synthetic samples.

\subsection{Identifying Unstable Systems}

In order to determine whether a given triple system is susceptible to the TEDI during its evolution, we make use of single and binary population synthesis stellar evolution codes (SSE and BSE, described in detail in Hurley et al. 2000, 2002), to which we couple an analytic calculation of the orbital evolution of the wider third component using Equation (2).

The specific procedure is as follows.

1. The inner binary in the triple is evolved using the BSE code up to a Hubble time (13.7 Gyrs), assuming Solar metallicity.

2. The third companion is evolved using the SSE code up to a Hubble time (13.7 Gyrs), assuming Solar metallicity.

3. The mass-dependent orbital evolution of the inner binary and outer single star are combined to form a time series evolution of all three stars. The inner orbit of the binary is determined by the BSE code. The outer orbit is updated at every output time step, $t_{i}$ of the BSE using Eq. (2), where the initial and final time steps are the preceding and current time steps

$$
\frac{a_{\text {out }}\left(t_{i}\right)}{a_{\text {out }}\left(t_{i-1}\right)}=\frac{m_{\text {triple }}\left(t_{i-1}\right)}{m_{\text {triple }}\left(t_{i}\right)} .
$$

We require that mass transfer between the inner binary and the third companion is always negligible, so that we may apply the adiabatic formula above. If the outer binary separation is smaller than $15 \mathrm{AU}$, we assume non-negligible mass transfer is possible either following a common-envelope phase for the inner-binary, or stellar evolution of the tertiary. We therefore consider all triples with outer separations smaller than 15 AU to be stable to the TEDI.

4. We calculate the stability coefficient, $Q$ at every step in the evolution, and using Eq. (1) find all systems that become unstable. Note that if the inner binary merges, or any of the stars explode as supernovae prior to destabilization, the system is considered stable (in the context of adiabatic mass loss). All other destabilized triples comprise our sample of unstable triples whose size is denoted by $N_{\text {des }}$.

5. For all destabilized systems we find the radius, $R_{\text {des }}$, of the mass losing evolving star at the point of destabilization, and note the types and radii of the other components. 
6. The fraction of destabilized systems is defined as $f_{\text {des }}=N_{\text {des }} / N_{\text {triple }}$.

\subsection{Observed Triples}

The observed triple sample (Tokovinin 2008) provides estimated masses and orbital periods for each star, but does not provide their mutual inclinations and eccentricities. We randomly assign each system relative inclinations and eccentricities drawn from realistic distributions (see Fabrycky \& Tremaine 2007).

We employ a distribution of eccentricities that is function of period, in line with observed binaries (Duquennoy \& Mayor 1991). For periods shorter than 1000 days, the eccentricity is chosen from a Rayleigh distribution $\left[d p \propto e \exp \left(-\beta e^{2}\right) d e\right]$ with $\left\langle e^{2}\right\rangle^{1 / 2}=\beta^{-1 / 2}=$ 0.33 . For periods longer than 1000 days, the eccentricity is chosen from an Ambartsumian distribution $(d p=2 e d e)$, which corresponds to a uniform distribution on the energy surface in phase space. The inclinations are chosen from a random distribution that is uniform in $\cos i$. We repeat the random sampling of these properties 30 times for each system.

We only evolve triples that are initially stable, since unstable systems are unlikely to be observable due to the instability timescale. If our random assignment of $e$ and $i$ produce an unstable system, we draw new values from the distributions.

We follow the procedure discussed in Section 3.1 to determine whether a given system becomes unstable. We find that the fraction of all observed triple systems with primary mass $m_{1}>1 \mathrm{M}_{\odot}$ destabilized within a Hubble time is $f_{\text {des,o }}=0.035 \pm 0.006$, where the error bars correspond to the $1 \sigma$ statistical uncertainty.

In Fig. 2] we illustrate the sensitivity of the results to the eccentricity distribution: we calculate the fraction of unstable systems for a complete grid of eccentricities. For this sample we conservatively assume retrograde orbits, $i=180^{\circ}$, which are the most stable configurations (see Eq. 1).

\subsection{Synthetic Sample of Triples}

We complement our use of the existing observed triple sample, which may introduce unknown biases, with a synthetic samples of triples. The method and the assumptions used to produce the synthetic samples are based on a method used by (Fabrvckv \& Tremaine 2007) for low mass triples and (Perets 2009) for high mass triples (with primary mass larger than $3 \mathrm{M}_{\odot}$ ).

We assume that the orbital and stellar characteristics of triple systems are equivalent to two (uncorrelated) binary systems, with the inner binary acting like a single mass when choosing the third component. The study by Kraus et al. (2011) suggests that this assumption is in good agreement with observations (though this was only checked for a small sample of triples). We choose initial orbital periods such that both the inner and outer binaries follow the observed distribution. The inner and outer periods are chosen from a distribution that is Gaussian for low mass binaries with $M<3 M_{\odot}$ (Raghavan et al. 2010) and log flat for massive binaries corresponding to $f(r) \propto 1 / r$ i.e., Öpik's law (Kobulnicky \& Frver 2007)

\footnotetext{
3 We assume A stars share a similar distribution as that of
}

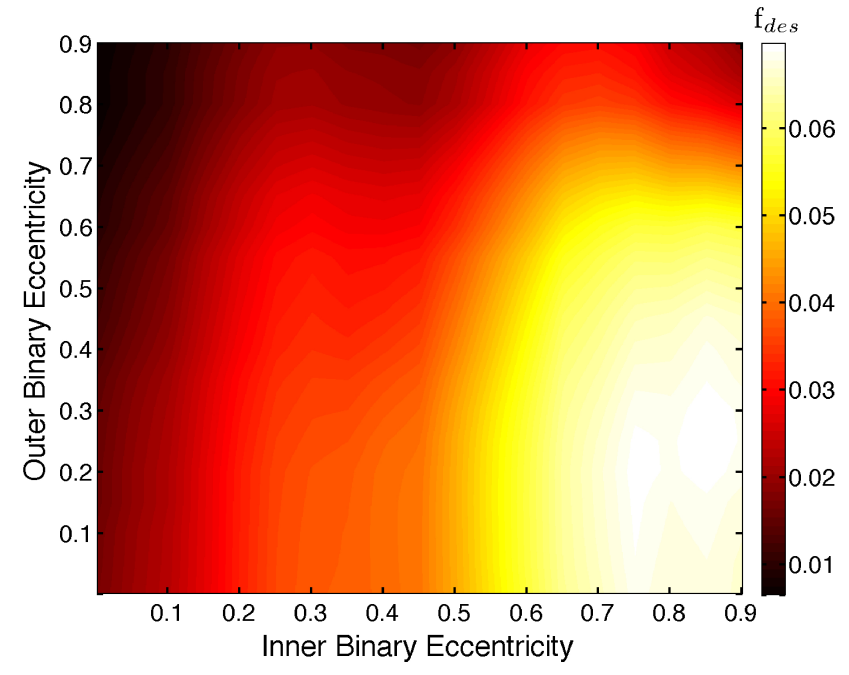

FIG. 2.- The fraction of destabilized systems, $f_{\text {des }}$, is shown for a grid of inner and outer eccentricities applied to the observed sample of triple systems (smoothed and interpolated; original bin size is $\Delta e=0.05)$.

The mass ratio of the inner binary, $q=m_{1} / m_{2}$, is chosen from a Gaussian distribution (mean of 0.6 and dispersion of 0.1) for low mass primaries and a power law for massive stars $\left(f(q) \propto q^{-0.4}\right)$;. The mass of the tertiary companion, $m_{3}$, is chosen such that $q=m_{3} /\left(m_{1}+m_{2}\right)$ follows the same mass ratio distribution. Thus the mass of the third star is correlated with the combined mass of the inner binary.

The inner and outer SMAs are computed from the masses and periods assuming non-interacting Keplerian orbits. The inclination and eccentricity distributions are chosen in the same manner as the observed sample. We produce a sample of triples with primary masses in the range $1 \leq m_{p} \leq 20$, with 1000 systems per primary mass bin (with bin size $\Delta M=0.3 \mathrm{M}_{\odot}$, for a total number of $6 \times 10^{4}$ systems). Note that since the triple outer component is randomly chosen from a distribution with respect to the inner binary mass, the term primary mass refers to the inner binary primary mass (which is not necessarily the most massive component in the triple).

In Fig. 3 $\mathrm{k}$ we show the fraction of destabilized triples from this sample as a function of primary mass. Fig. 3b shows the typical radius of the mass losing star at the time of destabilization $\left(R_{\mathrm{des}}\right)$. The change in the assumed binary properties between the low mass and the high mass stars introduces a sharp change in the typical $R_{\text {des }}$, since higher mass stars have more compact triple configurations on average, and can be destabilized at an earlier phase of their evolution, at which point the evolved star has a smaller radius.

Using the fraction of destabilized triples as a function of primary mass, $f_{\text {des,s }}(m)$, shown in Fig 3 , we can estimate the total fraction of destabilized triples. The statistical uncertainty for these fractions is small (1-2 $\%$; not shown). The fraction of observed stars in triple systems ranges from $11 \%$ for Solar like $(\mathrm{F}, \mathrm{G}, \mathrm{K})$ stars

F/G/K stars in the (Raghavan et al. 2010) sample. 

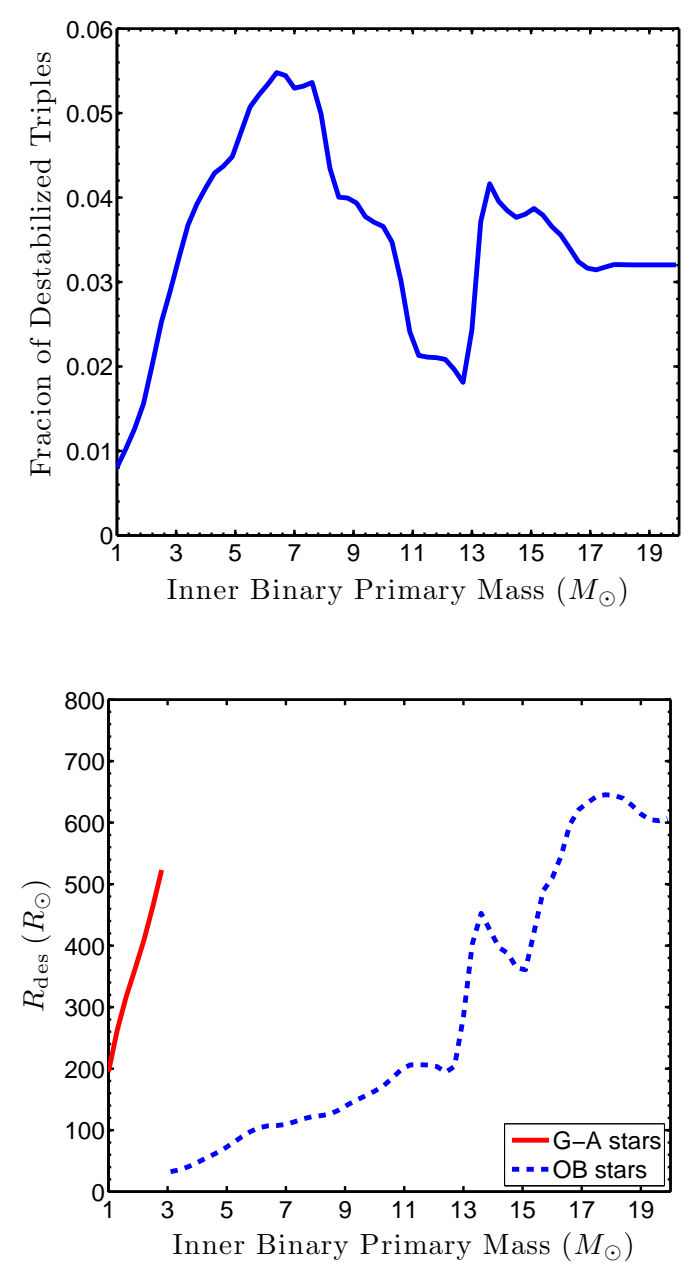

FIG. 3.- Top: The fraction of destabilized triples as a function of the inner binary primary component mass. The down turn at about $8 \mathrm{M}_{\odot}$ is due to supernova explosions that occur before the system can be destabilized due to mass loss. Bottom: The median radius of the mass losing stars at the onset of instability, as a function of mass. Separate lines indicate the low mass $(m<3$ $\left.\mathrm{M}_{\odot}\right)$ and the high mass stars $\left(m>3 \mathrm{M}_{\odot}\right)$, for which different triple distributions are assumed (see text).

(Raghavan et al. 2010; Rastegaev 2010) to possibly 50\% for more massive B stars (Evans 2011). We use a Salpeter initial mass function, $g(m)=d n / d m \propto m^{-2.35}$, and assume the triple fraction of massive stars is about 5 times larger than that of lower mass stars (Kobulnicky \& Fryer 2007; Evans 2011). Given these distributions, we estimate the total fraction of destabilized triple systems in our synthetic sample to be:

$$
\begin{gathered}
f_{\mathrm{des}, \mathrm{s}}=\int_{1}^{3} f_{\mathrm{des}, \mathrm{s}}(m) \frac{d n}{d m} d m \\
+5 \times \int_{3}^{30} f_{\mathrm{des}, \mathrm{s}}(m) \frac{d n}{d m} d m=0.010+0.043=0.053 .
\end{gathered}
$$

The overall fraction of destabilized triples in our synthetic sample is higher than but consistent with the results for the observed sample (0.053, compared with 0.035) (see also Fig. 2). Our estimates suggest that a few percent of all evolved triple systems undergo the TEDI.

We now turn to direct integrations of three-body systems to both validate the analytic instability criterion and to determine the fraction of unstable systems that produce a collision, $f_{\mathrm{col}}$.

\subsection{Three-body Simulations}

Because TEDI systems begin in stable configurations and adiabatically evolve into unstable configurations, they may not be directly comparable to unstable triples explored in previous studies (Valtonen \& Karttunen 2006). Therefore we conduct a series of three-body simulations including mass loss both to check that our destabilized systems are truly unstable, and to estimate the frequency of collisions based on the statistics of close approaches during the chaotic evolutionary phase. In the appendix, we provide a semi-analytic estimate of the collision rate.

The integrations are done using a modified version of the Hermite integrator described in Hut et al. (1995). The integrator uses a variable time step constrained to be $10^{-2}$ of the minimum collision time of any two stars. Reducing the time-step by a factor of 10 does not change the outcome. In runs without mass loss, energy is conserved to about one part in $10^{10}$ over the $10 \mathrm{Myr}$ run time. We only consider point masses, and include no tidal effects, or mass transfer. We use a constant mass loss rate, determined by the total mass loss in the system as found in the stellar evolution calculation, divided by a constant mass loss timescale, $\tau_{\text {loss }}=0.5 \mathrm{Myr}$ (the typical lifetime of the highly evolved stars, during which most of the mass loss occurs). After the mass loss epoch, $\tau_{\text {loss }}$, we continue the simulation, at constant mass up to 10 Myrs to check for longer term stability. In cases where our stellar evolution calculation shows that instability occurred only after both the primary and secondary in the inner binary evolved, we simulated the system beginning only at the second stage of mass loss (i.e. from the secondary), after the primary became a white dwarf.

We simulated 100 realizations of each observed triple system found to be unstable in Section 3, each realization differed only in the initial orbital phase of the stars. Since we do not account for mass transfer, we simulated only systems in which the inner binary separation was larger than $15 \mathrm{AU}$ (these comprise nearly half of the observed sample). We ran 300 different system configurations, resulting in a total of $3 \times 10^{4}$ runs.

To identify systems that become unstable, we require that either one star is ejected from the system, or an exchange occurs between the tertiary and one of the inner binary components. We find that approximately 0.33 of the systems found to be unstable in our simplified calculations become unstable in our simulations over a 0.5 Myr run. Although more triples are likely to show exchanges and escapes over longer time scales (for example we find approximately 55\% were destabilized within 10 Myrs), we adopt the short timescale simulation result, since our primary concern is with collisions, which are much more likely if they occur while the primary is on the AGB.

We take the most conservative estimate possible by assuming that the results from the three body integrations are more representative of the entire sample. We thus reduce the fraction of unstable observed systems found in Section $3\left(f_{\mathrm{des}, \mathrm{o}}=0.035\right)$ by a factor of 0.33 ; we adopt as the fraction of destabilized systems $f_{\text {des }}=0.035 \times 0.33 \simeq 0.012$. 


\section{STELLAR COLLISIONS DURING THE TEDI (CALCULATING $F_{\mathrm{COL}}$ )}

Having calculated the expected fraction of triple systems that are unstable to the TEDI, we now estimate the fraction of such systems that might suffer physical collisions. We show in the appendix that applying the results of previous simulations for both single star - binary encounters and unstable triple systems gives the fraction of unstable systems that undergo a collision as $0.49 \pm 0.03$. The analytically derived collision rate for unstable triples is significantly higher than that for random encounters because an unstable triple typically undergoes multiple close approaches before the system dissolves, and because the stellar radius at the time of collision is so large. Because the analytic results are based on initially unstable systems covering a limited part of the the three body problem phase-space, we determine a more conservative collision rate based on our three body integrations (see below).

\subsection{Collisions in three-body simulations}

We define collisions in our three body integrations as those cases where the closest approach between the primary and another star is smaller than the typical stellar radius of the evolving star, $R_{\text {des }}$ where $R_{\text {des }}$ is defined to be the stellar radius at the point of instability, when the system first satisfies $Q<Q_{\text {st }}$. We only classify close approaches as collisions if they occur within the $0.5 \mathrm{Myr}$ mass loss timescale when the star is likely bloated. Figure 4 shows the cumulative distribution of closest approaches amongst the unstable triples that we simulated. The distribution of radii of the evolved stars that go unstable ( $R_{\text {des }}$ defined before) is also shown. Most stars involved in the collisions are highly evolved AGB stars, with radii in the range of tens to hundreds of AU.

We find the collision rate between the evolving star and either of the other stellar components to be $f_{\text {col }} \simeq$ $0.09 \pm 0.03$ amongst the unstable systems, where the error bars are the $1 \sigma$ statistical uncertainty. Note that this fraction is smaller than that obtained from the simplified semi-analytic approximation discussed in the appendix. However, detailed analysis of the distribution of closest approaches between stars during the triple evolution, suggests that the simple linear approximation in Eq. A1 is inaccurate; we find that the closest approach statistics depend more complexly on the various orbital properties of the system. We therefore adopt the collision fraction found in our simulations for our subsequent calculations.

The sample chosen for the three body integrations excluded close inner binaries (with SMAs smaller than 15 $\mathrm{AU}$ ), because we did not account for mass transfer. Physical collisions are even more likely for these systems due to the monotonic dependence of the close approach probability on the inner binary separation (seen both in our integrations and in Eq. A1,) Thus the neglected systems likely have a higher collision probability. Because we adopt the same collision fraction for more compact triples, these rates should be considered a lower bound. Compact systems comprise about half of our observed sample, so the neglect of these systems in our calculations would change our results by at most a factor of two.

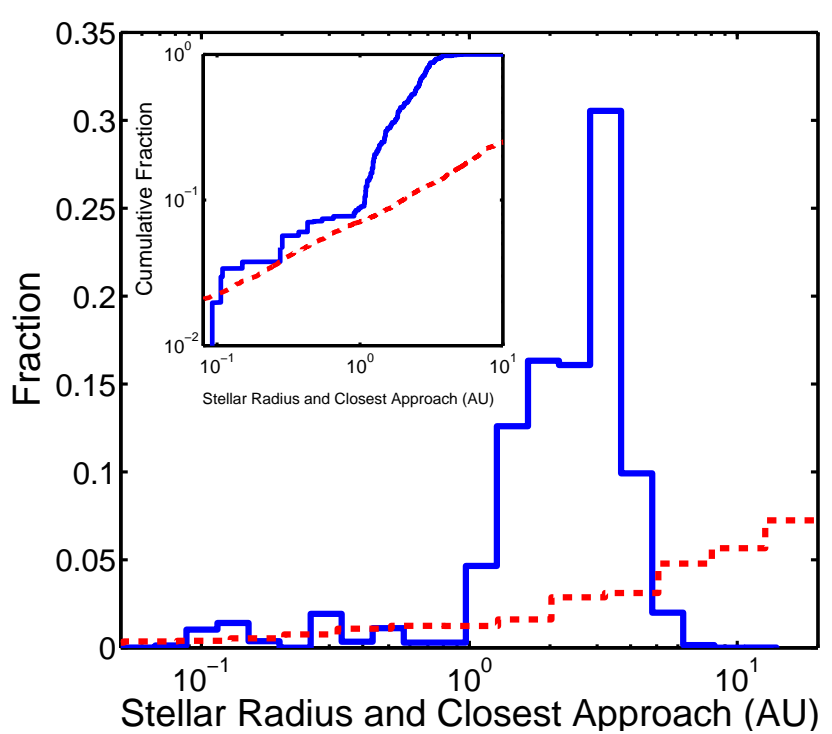

FIG. 4. - Stellar radii of evolved stars, and closest approaches between stars in destabilized systems. Dashed red lines indicate the distribution of closest approaches between the evolved star and another member of an evolving triple during the mass loss phase (taken to be $\tau_{\text {loss }}=0.5 \mathrm{Myr}$ ). Solid blue lines show the distribution of the stellar radii of the evolved mass losing star at the point of instability. Inset shows the cumulative distributions. Results were obtained from the three-body simulations of systems that became unstable.

Note that we cannot correctly follow the full evolution of systems in which tidal effects dominate the dynamics at any point. Nevertheless, for the short timescale of the TEDI, tidal effects become important when the closest approach is comparable to the stellar radius, and can be considered a sub-type of collision. Moreover, because most collisions occur when the physical radius is comparable to the tidal disruption radius, our neglect of tidal effects should not significantly alter our calculated collision rates. Determining the configurations of postcollision systems is beyond the scope of this work.

\section{GALACTIC COLLISION RATE DUE TO THE TEDI}

In the previous sections we provided estimates for the fraction of destabilized systems, $f_{\text {des }}$, and the fraction of colliding systems, $f_{\text {col }}$, among these systems. We can now make use of these results to estimate the overall rate of stellar collisions due to the TEDI. Taking the estimate of the collision rate together with the calculated fraction of destabilized triples, we find that the collision rate between evolved and companion stars/compact objects in a Milky Way like galaxy, over the age of the Universe, $t_{\mathrm{H}}$ is:

$$
\begin{aligned}
& \Gamma_{\text {col }}=\frac{N_{\star} \times f_{\text {evolve }} \times f_{\text {triple }} \times f_{\text {des }} \times f_{\text {col }}}{t_{\mathrm{H}}}= \\
& =1.2 \times 10^{-4}\left(\frac{N_{*}}{1.5 \times 10^{11}}\right) \\
& \quad \times\left(\frac{f_{\text {evolve }}}{0.1}\right)\left(\frac{f_{\text {triple }}}{0.1}\right)\left(\frac{f_{\text {des }}}{0.012}\right)\left(\frac{f_{\text {col }}}{0.09}\right) \mathrm{yr}^{-1},
\end{aligned}
$$

where $N_{\star}$ is the number of stars in the Galaxy (Binney \& Tremaine 2008; taking the mean stellar mass to be $0.3 \mathrm{M}_{\odot}$; Kroupa 2001), $f_{\text {evolve }}$ is the observed fraction of evolved stars (the fraction of WDs, 
Belczynski et al. 2002 can serve as a proxy for their total number), $f_{\text {triple }}$ is the fraction of triple systems (where we conservatively adopt the triple fraction of $f_{\text {triple }}=0.1$, found for $\mathrm{F} / \mathrm{G} / \mathrm{K}$ stars, Raghavan et al. 2010, for stars of all masses). We adopt $f_{\text {des }}$ (the fraction of triple systems that become unstable) and $f_{\text {col }}$ (the fraction of unstable systems in which a collision occurs) from our calculations in the previous sections.

For comparison, the collision rate in the GC M15, one of the densest in the galaxy $\left(n_{\mathrm{c}}>10^{6} \mathrm{M}_{\odot} \mathrm{pc}^{-3}\right.$; Umbreit et al. 2008), was estimated to be $\Gamma=2 \pm 1 \times 10^{-7}$ $\mathrm{yr}^{-1}$ (Umbreit et al. 2008; see also refs. Shara 1999; Lee et al. 2010). The total collision rate in Galactic GCs, which is dominated by such clusters (i.e. over 100 GCs exist in the Galaxy, but the dominant contribution to collsions come from the most dense, massive clusters $)$, is therefore $\Gamma_{\text {gal }}=4 \times 10^{-6}\left(N_{\mathrm{GC}} / 20\right) \mathrm{yr}^{-1}$, where $\mathrm{N}_{\mathrm{GC}} \approx 20$ is an estimate of the number of similar GCs in the galaxy as massive and dense as M15 (Gnedin \& Ostriker 1997). Collisions due to destabilized triples are therefore about $30\left(N_{\mathrm{GC}} / 20\right)$ times more frequent than collisions due to random encounters in GCs. The number of extragalactic GCs is typically proportional to the host galaxy mass (Brodie \& Strader 2006), suggesting a similar ratio of field to GC collisions in other galaxies.

Note that the collsion rate due to TEDI inside GCs scales with the total number of stars in GCs and does not depend on their specific density. Taking approximately 150 GCs exiting in the Galaxy with about $10^{6}$ stars in each cluster, we find the total collsion rate in GCs due to TEDI to be at most 0.1 percent of the total Galactic rate, and only about 3 percente of the collsion rate in GC due to random encounters. In other words, inside GCs, sellar collsions are dominated by random encounters rather than by triple stellar evolution.

\section{CONSEQUENCES OF THE TEDI}

The TEDI scenario has a wide variety of possible outcomes. Many of these are very similar to those resulting from binary-single and single-single close encounters between stars and/or compact objects that occur in globular clusters. However, the TEDI scenario provides a pathway to these exotic scenarios previously reserved for dense stellar populations.

In the following we discuss various outcomes of the TEDI and highlight their unique aspects, either in terms of their qualitative difference from typical encounters in clusters, or their specific relations to systems in the field. We first briefly overview several potential outcomes, and then discuss in more detail a specific example, providing a novel formation scenario for Sirius like eccentric WD binaries.

Stellar collisions in the field: As discussed above, the TEDI produces a high rate of stellar collisions in the field, most of which involve AGB stars; these were previously thought to comprise a negligible fraction of all collisions. These encounters might be observed as intermediate luminosity optical transients (with total energy ranging between $10^{46}-10^{48}$ ergs; these could be detectable in current and future optical transient surveys; Kulkarni \& Kasliwal 2009), possibly similar to those suggested to occur due to mergers or through tidal/off axis collisions in eccentric binaries (Kashi \& Soker 2010;
Smith 2011). Beside potentially producing immediate energetic transient events, the TEDI can produce a wide variety of collision products, including merged stars and blue stragglers as well as peculiar binaries.

Numerical simulations of close encounters with stars bloated to $100 R_{\odot}$ show that the resulting collisional and/or tidal captures lead to the formation of highly eccentric binaries (Bailey \& Davies 1999; Yamada et al. 2008). Such collisions might also lead to a common envelope phase and the formation of a close binary. The TEDI scenario therefore predicts the existence of a subpopulation of close, but eccentric, evolved binaries in the field. The TEDI can explain the puzzling orbits of WD binaries such as the nearby Sirius system (see below), as well as highly eccentric Barium binary systems (expected to form through mass transfer in a close binary). These systems pose a problem for standard binary stellar evolution models, which produce only circular close binaries (Bonačić Marinović et al.|2008; Karakas et al. 2000; Izzard et al. 2010) (but see Dermine et al. (2012), and references therein, for some alternative suggestions). The peculiar close WD-blue straggler binary (KOI-74) found by Kepler, which may have formed in a highly eccentric evolved binary system (Di Stefano 2011), could easily be produced by the TEDI. Similarly, TEDI evolution can lead to the formation of eccentric WD-WD binaries, which could provide unique sources for future gravitational wave detection missions, and were predicted to exist only in GCs (Willems et al. 2007). Our findings suggest that there could be a few times more such sources in the field than estimated to exist in GCs.

Neutron stars/black hole binaries: Mass loss from primaries more massive than $8 \mathrm{M}_{\odot}$ which undergo the TEDI, might form a close eccentric binary before the supernova explosion of the evolving massive star. Since such binaries have a higher probability of surviving the SN explosion (Hills 1983), the TEDI might aid in the retention of neutron stars and black holes in binaries, and the later formation of X-ray and pulsar binaries. Such triples could later evolve, during mass loss/mass transfer from the other triple components. Possible outcomes include instability and thus collisions with a neutron star/black hole, or even exchanges. See Freire et al. (2011) and Portegies Zwart et al. (2011) for the latter possibility, but note that these studies did not account for the possibility of a physical collision during the chaotic evolutionary phase.

Envelope stripping through collisions: A collision between a neutron star and its evolving companion could result in the tidal disruption of the latter. The remnants of this star might form an accretion disk surrounding the neutron star, which could provide the necessary mass and angular momentum to create a recycled pulsar (Verbunt et al. 1987). If the third companion is ejected during the course of the instability, the pulsar would appear as an isolated recycled pulsar in the field. If the third companion is retained it will be observed as a wide and likely eccentric recycled binary pulsar in the field. The latter scenario provides an additional channel for the formation of the pulsar J1903+0327 (Champion et al. 2008; Freire et al. 2011; Portegies Zwart et al. 2011). Another possibility is the formation of a recycled pulsar with a stripped companion on an eccentric orbit, if the companion's core stays 
intact.

There are many observed peculiar stars and compact remnants thought to form through envelope stripping by a close companion, such as hot sub-dwarf stars (Han et al. 2002), helium white dwarfs (Marsh et al. 1995), type Ib/c supernova progenitors (Yoon et al. 2010). However, cases are found where these peculiar objects are observed to be single or to have a wide companion, configurations that are inconsistent with the close binary progenitor scenario. Such cases might be explained through a TEDI induced collision. Further studies are required in order to quantitatively explore each scenario.

\section{FORMATION OF THE SIRIUS WD-BINARY SYSTEM}

One of the closest WD binaries to Earth is the Sirius system composed of a MS star of mass $2 M_{\odot}$ and a WD companion of about a Solar mass (Liebert et al. 2005). The SMA of $20 \mathrm{AU}$ and and high eccentricity of 0.59 make this binary quite peculiar, as standard stellar evolution scenarios suggest that the progenitor of such a close binary would have circularized during stellar evolution of the system (Bonačić Marinović et al. 2008; Izzard et al. 2010). However, the Sirius system could have formed through the TEDI; alternative scenarios are also in development (Dermine et al. 2012; P. Eggleton, private communication 2012).

To assess the likelihood that Sirius formed via this mechanism, we ran 6500 three-body simulations of the dynamical evolution of initially stable triple systems undergoing mass loss. For these systems, we follow the exact mass loss and radius evolution as obtained from the SSE code. The primary starts out with $M=5.05 M_{\odot}$, and ends as a $0.98 M_{\odot}$ WD. We evolve the systems analytically until the primary has reached about $4 \mathrm{M}_{\odot}$ and begins the rapid mass loss phase. We assume that the secondary and tertiary components have undergone no evolution. We explore a range of inner binary separations from $15-30$ AU, outer binary separations from $3.5-5.5$ times the inner separation, eccentricities from $0-0.25$, and companion masses from $0.6-5.5 M_{\odot}$. One component is always constrained to be $2 M_{\odot}$, the mass of Sirius A. The minimum separation of the inner binary is chosen to avoid mass transfer. To account for variations in the chaotic evolution at late times, we run each set of parameters starting from five different initial orbital phases. We limit the initial inclination of the systems to $i=0.1$ radian.

In Fig. 5] we show examples of final binary configurations of unstable systems where one of the stars was ejected and a Sirius like binary was left behind. Also shown is the observed SMA and eccentricity of the Sirius system. The Sirius binary falls within one of three populated regions of final configurations, suggesting that Sirius could have formed through the TEDI process. The inner cluster of eccentric systems is formed via ejection of the wider tertiary companion, while the outer cluster of eccentric systems formed via an exchange followed by the ejection of the initial inner binary companion to the WD. The moderately eccentric systems are composed of bound triple systems that underwent only mild eccentricity excitation.

We note that if Sirius and other objects formed through this mechanism, it might be possible to identify its ejected third star, as a very wide companion

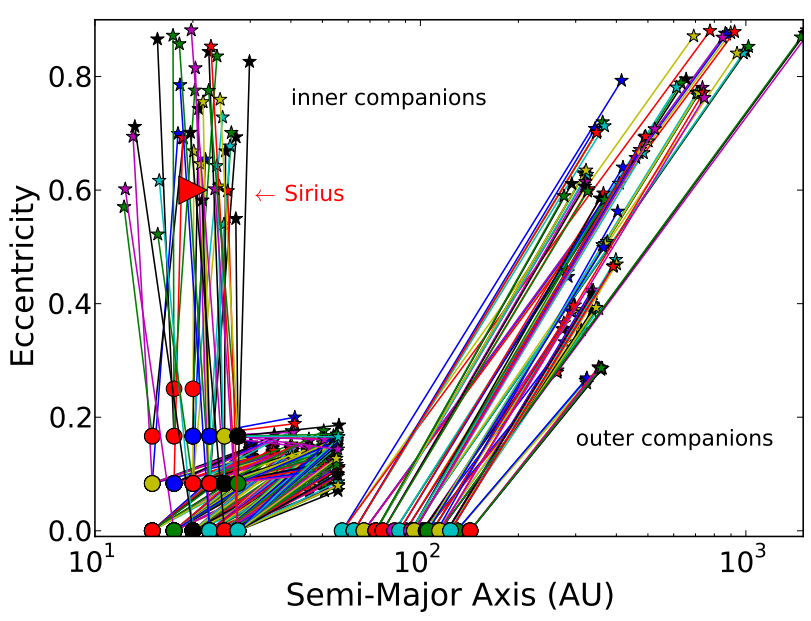

FIG. 5. - The initial and final eccentricities and semi-major axes of triple systems evolved using our three-body simulations. Circles represent the initial orbital configuration of a given pair of stars in the triple. Note that each circle corresponds not only to multiple realizations of a given system, but also to different masses for the third component. Lines connect the initial and final configurations, which are marked by stars. Each initial configuration can correspond to multiple final outcomes due to the chaotic nature of the instability. Final configurations similar to the observed Sirius system (marked with a red triangle), or even more eccentric are a typical outcome for systems with initial SMAs in the range 15$30 \mathrm{AU}$. Also shown are binaries formed via an exchange between the inner and outer companions, followed by ejection of the initial inner companion.

(e.g. Jiang \& Tremaine 2010) or through similar methods used to locate stars that originally formed close to the Sun (Portegies Zwart 2009; Bobylev et al. 2011).

\section{TRIPLE STELLAR EVOLUTION COUPLED WITH SECULAR KOZAI CYCLES}

Triple systems in which the inner and outer orbits have a high mutual inclination may secularly evolve through Kozai cycles, in which the inclination and eccentricity of the inner binary can periodically/quasiperiodically change by a large amplitude (Kozai 1962; Lidov 1962), and even flip from prograde to retrograde orbits (Naoz et al. 2011; and vice versa). It was already suggested that the coupling of secular Kozai evolution with dissipative processes such as tidal friction or GW emission could play an important role in the evolution of the inner binaries (e.g. Mazeh \& Shaham 1979; Eggleton \& Kisseleva-Eggleton 2006; Perets \& Fabrvcky 2009; Thompson 2011 and references therein). Mass loss evolution in triple systems could also couple with this secular Kozai process, and change its outcomes. Our simulations suggest that mass loss does not quench Kozai cyles. The high eccentricities that could be induced through the Kozai mechanism can therefore lead to close encounters between the components of the inner binary during pericenter approach prior to destabilization during the AGB stage. Kozai evolution could lead to close interactions between triple components at a late epoch in the evolution, even if the original triple did not interact while on the main sequence (see also Shappee \& Thompson 2012). The coupled stellar-dynamical evolution TEDI stable, Kozai susceptible, systems is beyond the scope of this paper, and will be explored elsewhere. 


\section{DISCUSSION AND SUMMARY}

In this paper we have studied the triple evolution dynamical instability (TEDI), in which mass loss in an evolving triple system leads to orbital instability. The chaotic evolution that ensues gives rise to close encounters between the stars and/or exchanges between them. Such evolution typically ends in a collision between two of the stars or the ejection of one of the components. This instability can lead to the dynamical formation of eccentric compact binaries, and produces a high stellar collision rate between stars in the field. The TEDI scenario results in a collision rate in the field of approximately $10^{-4} \mathrm{yr}^{-1}$ per Milky-Way Galaxy, about 30 times higher than the collision rate in all Galactic GCs. This high rate is attributable to two features of the TEDI. First, the chaotic orbital evolution results in binary-single star like resonant encounters in the field, which provide numerous opportunities for collisions. Secondly, the cross section for physical collisions is much larger than in typical random encounters. The mass loss that precipitates the instability reaches its maximum at the AGB phase, and therefore the instability typically occurs when the stellar radius is at its peak, a few hundred Solar radii (as seen in Fig. (4). The cross section for collisions is therefore increased proportionally. In contrast, random collisions in GCs are heavily dominated by MS and slightly evolved stars (Lombardi et al. 2006; Umbreit et al. 2008) with radii of $1-10$ Solar radii. The discrepancy between the peak collision phases is due to the $10^{5}-10^{6}$ year duration of the AGB phase. This timescale is a tiny fraction of a star's life, and thus short compared to the random encounter timescale, even in the densest environment. In contrast, the close encounters during the TEDI always coincide with the AGB phase. The TEDI model therefore requires not only a revision of the rate of stellar collisions, but also a reconsideration of their nature; most stellar collisions involve AGB stars.

In addition to enhancing the number of stellar collisions, the TEDI suggests that various types of exotic binaries collisionally formed inside GCs (X-ray binaries, cataclysmic variables, single and binary recycled pulsars, eccentric WD binaries, including eccentric inspiraling WD-WD GW sources, and stripped stars; Fabian et al. 1975; Poolev et al. 2003; Bailev \& Davies 1999; Lombardi et al. 2006; Willems et al. 2007 ) can similarly collisionally form through TEDI outside of dense clusters; adding an additional evolutionary channel for the formation of such binaries in the field. In particular, the TEDI provides a novel explanation for the origin of WD binaries observed in puzzling configurations, such as the well known Sirius system. The exotic stellar systems produced through these pathways have an outsize impact on our understanding of fundamental physics, and the evolution of stars in the field. They serve as the main sources for gravitational wave emission, and as unique probes of the structure of compact objects.

This study focused on the TEDI scenario for triple stellar systems. However, a system comprised of a stellar binary and a planet on a circumstellar orbit around an evolving star can also suffer a similar fate (Perets 2010, 2011). The evolution of such systems has recently been described by Kratter \& Perets (2012). In addition, the TEDI scenario can be extended to higher multiplicity systems (note that $1 / 4$ of all multiple systems are quadruples or higher order multiples Raghavan et al. 2010). Such systems can produce an even wider variety of outcomes than triple systems, and in general mimic almost any type of outcome that is expected to occur following close encounters in GCs.

We thank J. Hurley for providing the openly accessible SSE and BSE stellar evolution codes, S. Tremaine for helpful discussion on wide binaries in the context of this work, as well as F. Rasio and P. Eggelton for helpful comments on an earlier version of this manuscript. We also thank the referee, Christopher Tout, for helpful suggestions which much improved the clarity of this work. HBP acknowledges support from the BIKURA (FIRST) Israel Science Foundation and the CfA fellowship through the Harvard-Smithsonian Center for Astrophysics. KMK is supported by the Institute for Theory and Computation Fellowship through the Harvard College Observatory.

\section{APPENDIX}

\section{SIMPLIFIED ESTIMATE OF THE RATE OF STELLAR COLLISIONS IN THE TEDI SCENARIO}

The frequency of close approaches for initially unstable systems has been characterized in previous studies (see Saslaw et al. 1974; Valtonen \& Karttunen 2006 and references therein; see also Sigurdsson \& Phinney 1993 for the related issue of binary-single encounters). Triples starting from unstable configurations undergo similar evolution to that observed in simulations of low velocity, resonant encounters between a binary and a single star (Valtonen \& Karttunen 2006). The evolution of unstable triples can thus be modeled as a set of random encounters between an incoming third body and an inner binary. After any encounter, the incoming star can be ejected from the system, go through an exchange with one of the binary components, or collide with one of them. If a collision or ejection does not occur on a given passage, the cycle repeats, and the system experiences another encounter (though the components of the inner binary may change in the event of an exchange). These encounters cease when a collision or ejection occurs (a long-lasting stable triple can only be formed via energy dissipation, e.g. following a collision or a strong tidal encounter,). Previous three body integrations show that, on average, approximately 60 such encounters occur before a star is ejected (see Valtonen \& Karttunen 2006, and references therein), thus the probability for a close approach between any two stars in the system is proportionally increased compared with single-single stellar encounters. These results provide a simple estimate for the collision rate. During the chaotic evolution of unstable triple systems, the probability, $\mathrm{P}\left(<r_{\text {close }}, a_{\text {out }}\right)$ for a close approach of $r_{\text {close }} \ll a_{\text {out }}$ or smaller is (Valtonen \& Karttunen 2006):

$$
\mathrm{P}\left(<r_{\text {close }}, a_{\text {out }}\right) \sim 240\left[1+1.875\left(\frac{L / L_{\max }}{0.5}\right)^{2}\right] \times \frac{r_{\text {close }}}{a_{\text {out }}}
$$




$$
\simeq 0.11\left[1+1.875\left(\frac{L / L_{\max }}{0.5}\right)^{2}\right] \times\left(\frac{r_{\text {close }}}{100 \mathrm{R}_{\odot}}\right)\left(\frac{a_{\text {out }}}{1000 \mathrm{AU}}\right)^{-1},
$$

where $0 \leq \mathrm{L} / \mathrm{L}_{\max } \leq 1$ is the normalized angular momentum of the system $\left(L_{\max }\right.$ is the angular momentum for a circular orbit of the same energy). For the typical system that becomes unstable, Eq. (A1) gives a probability of order unity because the stars are often 100's of solar radii at the onset of instability; however, the time spent at this radius, $t_{\mathrm{R} \text {,des}}$, may be shorter than the typical length of the chaotic evolution, $t_{\text {chaotic }} \approx 60 \times p_{\text {out }}^{\text {des }}$, where $p_{\text {out }}^{\text {des }}$ is the period of the triple outer orbit at the point of destabilization. Thus the probability for a collision is quenched by a factor of approximately $\min \left(t_{\mathrm{R}, \mathrm{des}} / 60 p_{\mathrm{out}}^{\mathrm{des}}, 1\right)$.

Applying this correction factor, we can calculate the collision fraction for our observed sample. We take $R_{\text {des }}$ to be the stellar radius of the AGB star at the point of destabilization, $a_{\text {out }}$ to be the outer binary separation, and $t_{\mathrm{R} \text {,des }}$ to be the AGB lifetime at this radius or above. All these parameters are calculated separately for each system in our observed triples sample, at the point of instability, using the models described above (Section 3.1).

According to this calculation, the fraction of destabilized triples that suffer a direct collision between the evolving star and one of the other triple components is $0.49 \pm 0.03$ for the observed sample of triples (with the assumed eccentricity distribution). Comparison with our numerical results suggests that the analytic calculation significantly overestimates collisions for TEDI systems.

\section{REFERENCES}

Bailey, V. C. \& Davies, M. B. 1999, MNRAS, 308, 257

Belczynski, K., Kalogera, V., \& Bulik, T. 2002, ApJ, 572, 407

Binney, J. \& Tremaine, S. 2008, Galactic Dynamics: Second

Edition, ed. Binney, J. \& Tremaine, S. (Princeton University Press)

Bobylev, V. V., Bajkova, A. T., Mylläri, A., \& Valtonen, M. 2011, Astronomy Letters, 37, 550

Bonačić Marinović, A. A., Glebbeek, E., \& Pols, O. R. 2008, A\&A, 480, 797

Brodie, J. P. \& Strader, J. 2006, ARA\&A, 44, 193

Champion, D. J. et al. 2008, Science, 320, 1309

Davies, M. B. 2002, in Astronomical Society of the Pacific Conference Series, Vol. 265, Omega Centauri, A Unique Window into Astrophysics, ed. F. van Leeuwen, J. D. Hughes, \& G. Piotto, 215

Dermine, T., Izzard, R. G., Jorissen, A., \& Van Winckel, H. 2012 , arXiv:1203.6471

Di Stefano, R. 2011, AJ, 141, 142

Duquennoy, A. \& Mayor, M. 1991, A\&A, 248, 485

Eggleton, P. 2006, Evolutionary Processes in Binary and Multiple Stars, ed. Eggleton, P.

Eggleton, P. P. \& Kisseleva-Eggleton, L. 2006, Ap\&SS, 304, 75

Fabian, A. C., Pringle, J. E., \& Rees, M. J. 1975, MNRAS, 172, $15 \mathrm{P}$

Fabrycky, D. \& Tremaine, S. 2007, ApJ, 669, 1298

Freire, P. C. C. et al. 2011, MNRAS, 412, 2763

Gnedin, O. Y. \& Ostriker, J. P. 1997, ApJ, 474, 223

Hadjidemetriou, J. D. 1963, Icarus, 2, 440

Han, Z., Podsiadlowski, P., Maxted, P. F. L., Marsh, T. R., \& Ivanova, N. 2002, MNRAS, 336, 449

Heinämäki, P., Lehto, H. J., Valtonen, M. J., \& Chernin, A. D. 1999, MNRAS, 310, 811

Hills, J. G. 1983, ApJ, 267, 322

Hurley, J. R., Pols, O. R., \& Tout, C. A. 2000, MNRAS, 315, 543

Hurley, J. R., Tout, C. A., \& Pols, O. R. 2002, MNRAS, 329, 897

Hut, P., Makino, J., \& McMillan, S. 1995, ApJ, 443, L93

Iben, Jr., I. \& Tutukov, A. V. 1999, ApJ, 511, 324

Ivanov, A. V. \& Chernin, A. D. 1991, Pis ma Astronomicheskii Zhurnal, 17, 569

Ivanova, N. 2010, in American Institute of Physics Conference Series, Vol. 1314, American Institute of Physics Conference Series, ed. V. Kologera \& M. van der Sluys, 143-150

Izzard, R. G., Dermine, T., \& Church, R. P. 2010, A\&A, 523, $\mathrm{A} 10+$

Jiang, Y.-F. \& Tremaine, S. 2010, MNRAS, 401, 977

Karakas, A. I., Tout, C. A., \& Lattanzio, J. C. 2000, MNRAS, 316,689

Kashi, A. \& Soker, N. 2010, arXiv:1011.1222

Kiseleva, L. G., Eggleton, P. P., \& Orlov, V. V. 1994, MNRAS, 270,936

Kobulnicky, H. A. \& Fryer, C. L. 2007, ApJ, 670, 747

Kozai, Y. 1962, AJ, 67, 591

Kratter, K. M. \& Perets, H. B. 2012, Submitted
Kraus, A. L., Ireland, M. J., Martinache, F., \& Hillenbrand, L. A. 2011, ApJ, 731, 8

Kroupa, P. 2001, MNRAS, 322, 231

Kulkarni, S. \& Kasliwal, M. M. 2009, in Astrophysics with All-Sky X-Ray Observations, ed. N. Kawai, T. Mihara, M. Kohama, \& M. Suzuki, 312-+

Lee, W. H., Ramirez-Ruiz, E., \& van de Ven, G. 2010, ApJ, 720, 953

Lidov, M. L. 1962, Planet. Space Sci., 9, 719

Liebert, J., Young, P. A., Arnett, D., Holberg, J. B., \& Williams, K. A. 2005, ApJ, 630, L69

Lombardi, Jr., J. C., Proulx, Z. F., Dooley, K. L., Theriault, E. M., Ivanova, N., \& Rasio, F. A. 2006, ApJ, 640, 441

Mardling, R. A. 2008, in Lecture Notes in Physics, Berlin Springer Verlag, Vol. 760, The Cambridge N-Body Lectures, ed. S. J. Aarseth, C. A. Tout, \& R. A. Mardling, 59

Marsh, T. R., Dhillon, V. S., \& Duck, S. R. 1995, MNRAS, 275 828

Mazeh, T. \& Shaham, J. 1979, A\&A, 77, 145

Naoz, S., Farr, W. M., Lithwick, Y., Rasio, F. A., \& Teyssandier, J. 2011, Nature, 473, 187

Perets, H. B. 2009, ApJ, 698, 1330

-. 2010, arXiv:1001.0581

Perets, H. B. 2011, in American Institute of Physics Conference Series, Vol. 1331, American Institute of Physics Conference Series, ed. S. Schuh, H. Drechsel, \& U. Heber, 56-75

Perets, H. B. \& Fabrycky, D. C. 2009, ApJ, 697, 1048

Pooley, D. et al. 2003, ApJ, 591, L131

Portegies Zwart, S., van den Heuvel, E. P. J., van Leeuwen, J., \& Nelemans, G. 2011, ApJ, 734, 55

Portegies Zwart, S. F. 2009, ApJ, 696, L13

Raghavan, D., McAlister, H. A., Henry, T. J., Latham, D. W., Marcy, G. W., Mason, B. D., Gies, D. R., White, R. J., \& ten Brummelaar, T. A. 2010, ApJS, 190, 1

Rastegaev, D. A. 2010, AJ, 140, 2013

Evans, N. R. 2011, arXiv:1102.5316

Saslaw, W. C., Valtonen, M. J., \& Aarseth, S. J. 1974, ApJ, 190, 253

Shappee, B. J. \& Thompson, T. A. 2012, arXiv:1204.1053

Shara, M. M. 1999, Phys. Rep., 311, 363

Shara, M. M. 2002, Stellar Collisions, Mergers and Their Consequences, ed. Shara, Michael M. (Astronomical Society of the Pacific Conference Series)

Sigurdsson, S. \& Phinney, E. S. 1993, ApJ, 415, 631

Smith, N. 2011, MNRAS, 567

Thompson, T. A. 2011, ApJ, 741, 82

Tokovinin, A. 2008, MNRAS, 389, 925

Umbreit, S., Chatterjee, S., \& Rasio, F. A. 2008, ApJ, 680, L113

Valtonen, M. \& Karttunen, H. 2006, The Three-Body Problem, ed. Valtonen, M. \& Karttunen, H.

Valtonen, M., Mylläri, A., Orlov, V., \& Rubinov, A. 2008, in IAU Symposium, Vol. 246, IAU Symposium, ed. E. Vesperini, M. Giersz, \& A. Sills, 209-217 
Verbunt, F., van den Heuvel, E. P. J., van Paradijs, J., \& Rappaport, S. A. 1987, Nature, 329, 312

Willems, B., Kalogera, V., Vecchio, A., Ivanova, N., Rasio, F. A., Fregeau, J. M., \& Belczynski, K. 2007, ApJ, 665, L59
Yamada, S., Okazaki, A. T., \& Fujimoto, M. Y. 2008, ApJ, 678, 922

Yoon, S.-C., Woosley, S. E., \& Langer, N. 2010, ApJ, 725, 940 